\title{
FISICA ALL'ENEM E AL CORSO DI CHIMICA TECNICA PRESSO L'ISTITUTO FEDERALE DI AMAPÁ (IFAP): UN CONFRONTO CURRICULARE
}

\section{ARTICOLO ORIGINALE}

ARMO, Denny Rodrigues do ${ }^{1}$, BASTOS, Argemiro Midonês ${ }^{2}$, FECURY, Amanda Alves $^{3}$, DENDASCK, Carla Viana ${ }^{4}$, OLIVEIRA, Euzébio de ${ }^{5}$, DIAS, Claudio Alberto Gellis de Mattos $^{6}$

CARMO, Denny Rodrigues do. Et al. Fisica all'ENEM e al corso di chimica tecnica presso I'Istituto federale di Amapá (IFAP): un confronto curriculare. Revista Científica Multidisciplinar Núcleo do Conhecimento. Anno 06, Ed.03, Vol.03, pagg. 80-88. Marzo 2021. ISSN: 2448-0959, Link di accesso: https://www.nucleodoconhecimento.com.br/formazione-it/fisica-allenem, DOI: 10.32749/nucleodoconhecimento.com.br/formazione-it/fisica-allenem

\footnotetext{
${ }^{1}$ Studente del corso tecnico di chimica (scuola superiore) presso l'Istituto di educazione di base, tecnica e tecnologica di Amapá (IFAP).

${ }^{2}$ Fisico, PhD in Biodiversità e Biotecnologie (UFPA), Professore e ricercatore del Corso di Laurea in Fisica presso I'Istituto di Base, Tecnico e Tecnologico di Amapá (IFAP) e del Corso di Laurea in Educazione Professionale e Tecnologica (PROFEPT IFAP).

${ }^{3}$ Biomedico, PhD in Malattie topiche, Professore e ricercatore del Corso di Medicina al Campus Macapá, Università Federale di Amapá (UNIFAP).

${ }^{4}$ Teologo, PhD in Psicoanalisi Clinica. Ha lavorato per 15 anni con la Metodologia Scientifica (Metodo di Ricerca) nell'Orientamento alla Produzione Scientifica di studenti di Master e Dottorato di Ricerca. Specialista in Ricerche di Mercato e Ricerche focalizzate sull'area Salute.

${ }^{5}$ Biologo, PhD in Malattie topiche, Professore e ricercatore del Corso di Educazione Fisica presso l'Università Federale del Pará (UFPA).Biologo, PhD in Malattie topiche, Professore e ricercatore del Corso di Educazione Fisica presso l'Università Federale del Pará (UFPA).

${ }^{6}$ Biologo, dottore in teoria e ricerca del comportamento, professore e ricercatore del corso di laurea in chimica presso l'Istituto di educazione di base, tecnica e tecnologica di Amapá (IFAP) e il programma di laurea in educazione professionale e tecnologica (PROFEPT IFAP).
}

$\mathrm{RC}: 78536$

Disponível em: https://www.nucleodoconhecimento.com.br/formazione-it/fisica- 


\section{ASTRATTO}

II National High School Examination (ENEM) è uno strumento valutativo e selettivo per consentire agli studenti di accedere all'istruzione superiore. Gli Istituti Federali di Educazione, Scienza e Tecnologia (IFs) sono istituzioni create dal governo federale con l'obiettivo di formare professionisti competenti. Lo scopo di questo studio è confrontare il contenuto delle domande di fisica dell'esame di scuola superiore nazionale (ENEM) tra gli anni 2014-2018 con il contenuto del curriculum del corso di chimica tecnica presso l'Istituto federale di Amapá (IFAP). L'insegnamento di fisica nel corso tecnico di Chimica dell'IFAP non presenta una divisione che dia la priorità alle materie più presenti in ENEM. ENEM di solito contestualizza le sue domande. Questa potrebbe essere una pratica comune nella fisica delle scuole superiori, in quanto aiuterebbe a una migliore comprensione. Inoltre, è necessario non frammentare il contenuto durante l'insegnamento, né come la sua composizione con altre materie. II corso tecnico all'IFAP non sarebbe il luogo di studio appropriato per coloro che vogliono solo finire il liceo. II contenuto va oltre quanto richiesto, ma con ritagli adeguati alla parte tecnica, comprese le pratiche di laboratorio e le discipline strettamente tecniche. L'assenza di interdisciplinarietà e contestualizzazione rende difficile l'assorbimento del contenuto, formando studenti con difficoltà a pensare alla fisica.

Parole chiave: ENEM, EPT, Insegnamento, Fisica, IFAP.

\section{INTRODUZIONE}

Creato dal governo federale del Brasile nel 1998, il National High School Exam (ENEM) mirava a valutare gli studenti che hanno completato la scuola superiore. Nel tempo, ENEM ha acquisito maggiore rilevanza quando è stato utilizzato non solo come strumento di valutazione, ma anche come strumento selettivo per l'ingresso degli studenti nell'istruzione superiore (Silveira et al., 2015).

RC: 78536

Disponível em: https://www.nucleodoconhecimento.com.br/formazione-it/fisica- 
Nel test ENEM, l'argomento della fisica viene affrontato insieme alle materie di biologia e chimica nell'area della conoscenza della scienza della natura. Anche se non esiste una separazione formale, è possibile vedere che le questioni di Scienze Naturali possono essere organizzate tra le tre discipline analizzando i contenuti trattati. (Silveira et al., 2015).

Gli Istituti Federali di Educazione, Scienza e Tecnologia sono istituzioni create dal governo federale con l'obiettivo di formare professionisti competenti. Questi possono presentare diversi corsi come lauree, master o dottorati, ma la modalità principale è l'istruzione tecnica, principalmente l'istruzione tecnica integrata con la scuola superiore (Pacheco, 2010).

In Brasile, tutti gli stati hanno almeno un Istituto Federale, alcuni ne hanno anche più di uno, per un totale di 38 unità. Un istituto federale può essere suddiviso in campus, con un totale attuale di 644 in funzione. (Brasil, 2018).

L'Istituto Federale di Istruzione, Scienza e Tecnologia dello Stato di Amapá (IFAP) è stato creato il 28 dicembre 2008 con la legge n. 11.892 (Marques et al., 2020) e mira a contribuire allo sviluppo dello stato (Castro et al., 2020). IFAP offre corsi che vanno dall'istruzione superiore alla scuola superiore, e con ciò riesce a raggiungere un vasto pubblico a diversi livelli, si compone anche di diversi campus, tra cui possiamo citare il campus Macapá, situato nella capitale (Brasil, 2019; Penha et al., 2020).

Il campus Macapá offre il corso tecnico di chimica della scuola superiore in una forma integrata. In questo corso abbiamo le componenti curriculari della National Common Base riferite alle aree delle lingue, della matematica, delle scienze umane e delle scienze naturali. Nell'area delle scienze naturali, abbiamo la componente curriculare della fisica che viene coperta durante i tre anni del corso. Ogni anno ha un carico di lavoro di 80 ore all'anno ei contenuti insegnati sono organizzati in quattro unità, per un totale di 12 unità nel triennio. (Brasil, 2016).

$\mathrm{RC}: 78536$

Disponível em: https://www.nucleodoconhecimento.com.br/formazione-it/fisica- 
Presso l'Istituto Federale di Amapá (IFAP) le materie del menu della componente "Fisica" del primo anno del corso tecnico integrato integrale sono: Introduzione a Fisica e Cinematica; Dinamica; Lavoro ed energia (che include anche Quantità di movimento e Impulso); Gravitazione e statica dei fluidi. Nel secondo anno, il curriculum comprende: calore e temperatura; Termodinamica; Ottica geometrica; Oscillazioni e onde (che include anche il movimento armonico semplice). Nel terzo e ultimo anno i contenuti sono: Elettrostatico; Elettrodinamica; Magnetismo; ed Elettromagnetismo (Brasil, 2016).

All'interno della matrice del curriculum Enem, ci sono competenze e oggetti di conoscenza relativi a queste competenze. Gli oggetti di conoscenza relativi alla fisica sono raccolti all'interno di 7 argomenti: conoscenza di base e fondamentale (che include argomenti di analisi dimensionale e sistemi di unità); movimento, equilibrio e scoperta delle leggi fisiche (che include cinematica, dinamica, idrostatica, statica, impulso e quantità di movimento); energia, lavoro e potere (che include questioni relative al lavoro e all'energia); la meccanica e il funzionamento dell'universo (che include argomenti legati alla gravitazione); fenomeni elettrici e magnetici (con argomenti relativi a Elettricità e Magnetismo); oscillazioni, onde, ottiche e radiazioni (che include soggetti da Ottica a Onda); e calore e fenomeni termici (che include argomenti legati alla termologia) (Brasil, 2015).

\section{OBIETTIVI}

Confronta il contenuto delle domande di fisica dell'esame di scuola superiore nazionale (ENEM) tra gli anni 2014 e 2018 con il contenuto curriculare del corso di chimica tecnica presso l'Istituto federale di Amapá (IFAP).

\section{MATERIALE E METODI}

La ricerca è stata condotta utilizzando domande del National High School Examination (ENEM) tratte dal programma (software) Super professor, acquisito

RC: 78536

Disponível em: https://www.nucleodoconhecimento.com.br/formazione-it/fisica- 
dalla

società

Interbits

Informática

(https://www.sprweb.com.br/mod_superpro/index.php ). Nel programma sono state selezionate la disciplina fisica e gli anni dal 2014 al 2018. Le domande sono state classificate in base al programma (previa analisi e rimozione delle domande sovrapposte). Successivamente, è stato effettuato un confronto tra questi e il programma della componente fisica del corso tecnico dell'Istituto Federale di Amapá (IFAP). La ricerca bibliografica è stata condotta su articoli scientifici, sui computer dell'Istituto presso l'Istituto Federale di Educazione, Scienza e Tecnologia di Amapá, Campus Macapá, situato a Rodovia BR 210 KM 3, s / n - Bairro Brasil Novo. CEP: 68.909-398, Macapá, Amapá, Brasile. I dati sono stati compilati nell'applicazione Excel, parte della suite Office di Microsoft Corporation.

\section{RISULTATI}

La classificazione del contenuto sovrapposto delle domande di fisica ENEM tra il 2014 e il 2018, per quantità e percentuale di domande, appare nella tabella 1. Tre (3) soggetti non presentano alcuna domanda (analisi dimensionale / sistema di unità, gravitazione e fisica moderna). La maggior parte dei soggetti ha una (1) o due (2) domande. Elettricità + Magnetismo e Cinematica presentano 4 domande ciascuno. Sei (6) delle domande riguardano il tema del lavoro e dell'energia nonché il tema dell'ottica. L'argomento Wave comprende un totale di 17 domande.

$\mathrm{RC}: 78536$

Disponível em: https://www.nucleodoconhecimento.com.br/formazione-it/fisica- 
Tabella 1. Classificazione del contenuto sovrapposto delle domande di fisica ENEM tra il 2014 e il 2018, per quantità e percentuale di domande.

\begin{tabular}{l|c|c}
\hline \multicolumn{3}{c}{ Classificação do autor após análise de conteúdo sobreposto } \\
\hline \multicolumn{1}{c|}{ Conteúdo de Física } & Quantidade & Porcentagem \\
\hline Análise Dimensional / Sistemas de Unidades & 0 & $0 \%$ \\
\hline Gravitação & 0 & $0 \%$ \\
\hline Moderna & 0 & $0 \%$ \\
\hline Cinemática + Impulso e Quantidade de Movimento & 1 & $2 \%$ \\
\hline Dinâmica + Impulso e Quantidade de Movimento & 1 & $2 \%$ \\
\hline Hidrodinâmica & 1 & $2 \%$ \\
\hline Hidrostática & 1 & $2 \%$ \\
\hline Trabalho e Energia + Movimento Harmônico & 1 & $2 \%$ \\
\hline Temática + Trabalho e energia & 1 & $2 \%$ \\
\hline Tematica & 1 & $2 \%$ \\
\hline Cinemática + Dinâmica & 2 & $3 \%$ \\
\hline Dinâmica & 2 & $3 \%$ \\
\hline Estática & 2 & $3 \%$ \\
\hline Impulso e Quantidade de Movimento & 2 & $3 \%$ \\
\hline Trabalho e Energia + termologia & 2 & $3 \%$ \\
\hline Eletricidade + Magnetismo & 4 & $7 \%$ \\
\hline Cinemática & 4 & $7 \%$ \\
\hline Trabalho e Energia & 6 & $10 \%$ \\
\hline Óptica & 6 & $10 \%$ \\
\hline Termologia & 7 & $11 \%$ \\
\hline Ondulatória & 17 & $28 \%$ \\
\hline & $\mathbf{6 1}$ & $\mathbf{1 0 0} \%$ \\
\hline
\end{tabular}

La tabella 2 mostra gli argomenti del menu di fisica del corso tecnico di chimica all'IFAP, mostrando il numero di unità (per bimestre) e l'anno coperto. Le unità da I a IV devono essere insegnate in ottanta (80) ore di lezione. Nel primo anno vengono normalmente utilizzate 12 ore per insegnare l'unità I, 28 ore per insegnare l'unità II, 20 ore per l'unità III e 20 ore per l'unità IV. Nel secondo anno, l'unità I viene solitamente insegnata in 20 ore, così come ciascuna delle unità successive (II, III e

RC: 78536

Disponível em: https://www.nucleodoconhecimento.com.br/formazione-it/fisicaallenem 
IV). Nel terzo anno, l'unità I è completata in 16 ore, l'unità II in 36 ore, l'unità III in 14 ore e l'unità IV in 14 ore.

Tabella 2. Materie del menu di fisica del corso tecnico di chimica all'IFAP, per anno e unità.

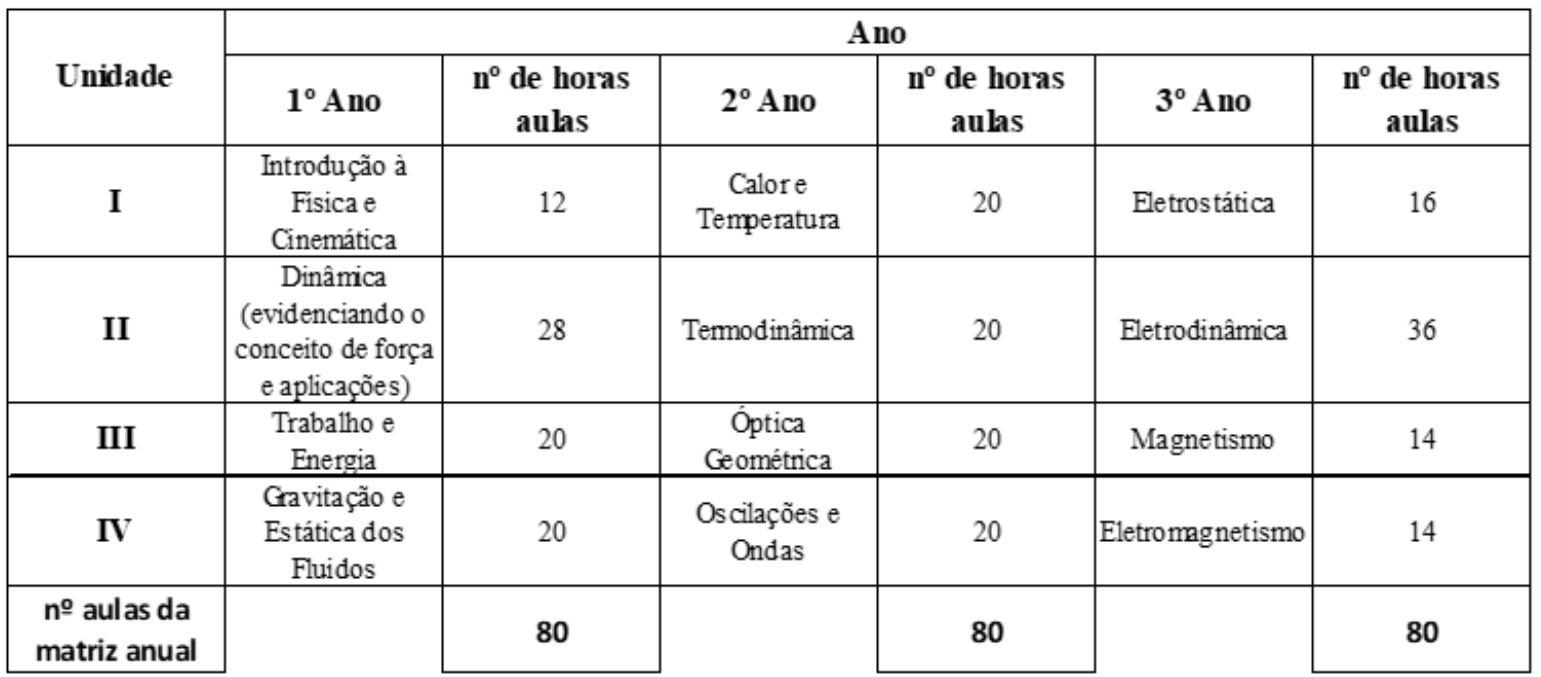

La classificazione semplificata del contenuto delle domande di fisica ENEM tra il 2014 e il 2018, per quantità e percentuale di domande, è mostrata nella tabella 3. L'argomento Tematico compare nel $2 \%$ delle domande, seguito dall'argomento Elettricità + Magnestimo (7\%) , ottica (10\%), termologia (11\%); Onda $(28 \%)$ e Meccanica (43\%).

RC: 78536

Disponível em: https://www.nucleodoconhecimento.com.br/formazione-it/fisica- 
Tabella 3. Classificazione semplificata del contenuto delle domande di fisica ENEM tra il 2014 e il 2018, per quantità e percentuale di domande.

\begin{tabular}{l|c|c|}
\hline Assunto & Quantidade & Porcentagem \\
\hline Tematica & 1 & $2 \%$ \\
\hline Eletricidade + Magnetismo & 4 & $7 \%$ \\
\hline Óptica & 6 & $10 \%$ \\
\hline Termologia & 7 & $11 \%$ \\
\hline Ondulatória & 17 & $28 \%$ \\
\hline Mecânica & 26 & $43 \%$ \\
\hline Total & $\mathbf{6 1}$ & $\mathbf{1 0 0 \%}$ \\
\hline
\end{tabular}

La classificazione semplificata del contenuto delle domande di fisica ENEM tra il 2014 e il 2018, diviso per anno in cui vengono insegnate nel corso tecnico di chimica dell'IFAP è riportata nella tabella 4. La percentuale mostrata si riferisce alla porzione di domande ENEM all'interno ogni soggetto.

Tabella 4. Classificazione semplificata del contenuto delle domande di fisica ENEM tra il 2014 e il 2018, diviso per anno in cui vengono insegnate nel corso tecnico di chimica

dell'IFAP

\begin{tabular}{|c|c|c|c|c|}
\hline & \multicolumn{4}{|c|}{ Curso técnico em química do IFAP } \\
\hline & $1^{\circ}$ & $2^{\circ}$ & $3^{a}$ & Todos \\
\hline \multirow{3}{*}{$\begin{array}{c}\text { Assuntos com } \\
\text { as porcentagem } \\
\text { de questões }\end{array}$} & \multirow{3}{*}{ Mecânica (43\%) } & Óptica $(10 \%)$ & \multirow{3}{*}{$\begin{array}{c}\text { Eletricidade }+ \\
\text { Magnestismo (7\%) }\end{array}$} & \multirow{3}{*}{ Tematica $(2 \%)$} \\
\hline & & Termologia (11\%) & & \\
\hline & & Ondulatória (28\%) & & \\
\hline $\begin{array}{c}\text { Porcentagem } \\
\text { Total }\end{array}$ & $43 \%$ & $49 \%$ & $7 \%$ & $100 \%$ \\
\hline
\end{tabular}

La figura 1 mostra il numero di domande di fisica all'ENEM tra il 2014 e il 2018 in base al grado di difficoltà. Le domande classificate dal programma Super professor

RC: 78536

Disponível em: https://www.nucleodoconhecimento.com.br/formazione-it/fisica- 
come di bassa difficoltà sono diminuite in quantità dal 2014 al 2016, aumentando nuovamente dal 2016 al 2018. Quelle di media difficoltà sono aumentate dal 2014 al 2016, e sono diminuite dal 2016 al 2018. Quelle considerate di alta difficoltà compaiono solo nel 2015.

Figura 1 Numero di domande di fisica all'ENEM tra il 2014 e il 2018 in base al grado di difficoltà

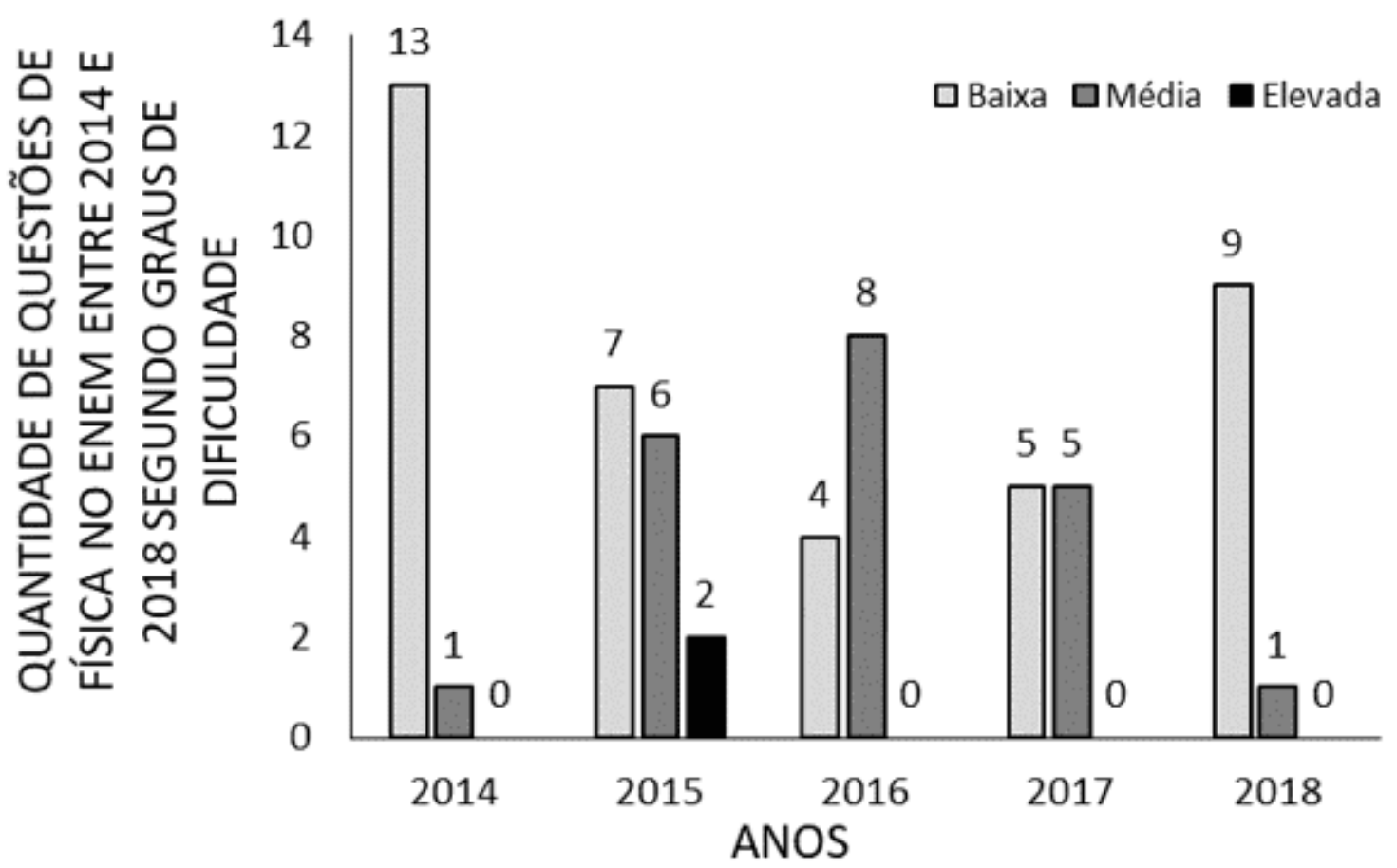

\section{DISCUSSIONE}

II National High School Exam ha come parametro di contestualizzare le domande dell'esame fisico collegandole alla vita quotidiana delle persone (Silveira et al., 2014). In questo senso, la differenza presentata tra i soggetti della valutazione fisica nel periodo studiato (tabella 1) potrebbe essersi verificata come conseguenza di questa contestualizzazione. In uno studio sulla presenza della fisica nella vita quotidiana degli studenti lavoratori, sono state rilevate domande sull'uso di diverse energie

RC: 78536

Disponível em: https://www.nucleodoconhecimento.com.br/formazione-it/fisica- 
(elettriche, chimiche), in situazioni come impianti di produzione di alcol e luoghi per l'installazione di apparecchiature audio nelle automobili (Toti e Pierson, 2010). Anche l'ottica appare regolarmente nella vita delle persone. Lo studio della luce può aiutare un ingegnere a sapere quanta ombra il suo edificio progettato creerà nel quartiere; o come una tazza o un oggetto metallico, leggi un'immagine; o anche perché il cielo è azzurro quando il giorno è soleggiato (Ribeiro e Verdeaux, 2012).

Nel corso tecnico, come parte dell'elettromagnetismo, c'è un'introduzione alla relatività speciale, che è un argomento della fisica moderna (Brasil, 2016). La fisica moderna al liceo non è ancora un contenuto consolidato e non c'è consenso sul suo insegnamento. Probabilmente per questo motivo l'assenza di questo argomento in ENEM (Silva et al., 2013).

Di solito i curricula dividono l'anno scolastico delle superiori in quattro bimestrali. Ogni bimestre corrisponde a un'unità, di 20 ore ciascuna. In un anno con 80 ore, la divisione sarebbe quindi equa. Tuttavia, l'insegnamento di alcune materie richiede più tempo di altre (tabella 2). La differenza tra il tempo pianificato e il tempo reale per ciascuna unità è probabilmente dovuta alla programmazione frammentata del contenuto. In questo modo, l'apprendimento diventa libero e il contenuto presentato, frammentato, presenta diversi fattori di difficoltà, che richiedono meno o più ore per il suo completamento (Gaspar, 1997). Inoltre, il tempo di lezione in una classe tradizionale viene dedicato anche ad altri compiti (chiamata, appunti) e non all'insegnamento della disciplina stessa (Pires e Veit, 2006).

Nella matrice di riferimento di Enem, la maggior parte degli oggetti di studio sono legati alla meccanica. In questa stessa matrice, i soggetti dell'onda, dell'ottica, dell'elettromagnetismo e della termodinamica hanno un numero simile di oggetti. Ma la presenza di questi soggetti nelle domande non è simile. Solo la meccanica è molto presente sia in questa matrice che nelle domande (tabella 3). La matrice di riferimento comprende, nell'argomento di meccanica, molti concetti diversi, che non si verificano con altri soggetti, ad esempio, l'onda. Forse questa divisione praticata

$\mathrm{RC}: 78536$

Disponível em: https://www.nucleodoconhecimento.com.br/formazione-it/fisica- 
dall'ENEM e diversa dalla divisione del liceo tecnico, causa la differenza percentuale osservata (Brasil, 2015).

Alcuni contenuti insegnati nella componente fisica all'interno del corso tecnico di chimica all'IFAP hanno, ad esempio, una carica di 80 ore (elettricità + magnetismo) e corrispondono al $7 \%$ del contenuto caricato in ENEM. Altri, come la termologia, vengono insegnati nell'istruzione tecnica in 40 ore e corrispondono all'11\% dell'esame nazionale. Altri ancora, come un'onda, rappresentano il $28 \%$ della valutazione ENEM e vengono insegnati in 20 ore. Questa disparità si verifica probabilmente perché il ruolo fondamentale dell'IFAP, nel suddetto corso, è quello di formare tecnici in chimica (Brasil, 2019). Ciò non significa che gli studenti che completano l'istruzione secondaria tecnica non prendano l'ENEM per accedere all'istruzione superiore.

L'insegnamento della fisica presenta ancora difficoltà per quanto riguarda l'assorbimento della conoscenza da parte degli studenti, il che significa che la maggior parte degli studenti non presenta prestazioni soddisfacenti in questa disciplina (Sbf, 2011; Barroso et al., 2018).

II test ENEM dovrebbe essere coerente con ciò che lo studente ha imparato al liceo (normale o tecnico). È stato possibile vedere che la difficoltà di ENEM è cambiata nel corso degli anni (figura 1). II tentativo di aumentare la difficoltà delle domande d'esame nazionali (2014-2016) è stato annullato nei due anni successivi. Probabilmente poiché l'insegnamento della fisica a livello secondario ha ancora una bassa performance di assorbimento della conoscenza, forse questo ha influenzato la decisione di abbassare il livello di difficoltà di questa componente negli esami 2017 e 2018 (Barroso et al., 2018).

$\mathrm{RC}: 78536$

Disponível em: https://www.nucleodoconhecimento.com.br/formazione-it/fisica- 


\section{CONCLUSIONE}

L'insegnamento di fisica nel corso tecnico in Chimica presso IFAP non presenta una divisione che privilegi le materie maggiormente presenti in ENEM.

ENEM di solito contestualizza le sue domande. Questa potrebbe essere una pratica comune nella fisica delle scuole superiori, in quanto aiuterebbe a una migliore comprensione. Inoltre, è necessario non frammentare il contenuto durante l'insegnamento, o come sua composizione con altre materie.

Il corso tecnico IFAP non sarebbe il luogo di studio appropriato per coloro che vogliono solo finire il liceo. II contenuto va oltre quanto richiesto, ma con ritagli adeguati alla parte tecnica, comprese le pratiche di laboratorio e le discipline strettamente tecniche.

L'assenza di interdisciplinarietà e contestualizzazione rende difficile l'assorbimento del contenuto, formando studenti con difficoltà a pensare alla fisica.

\section{RIFERIMENTI}

BARROSO, M. F.; RUBINI, G.; SILVA, T. Dificuldades na aprendizagem de Física sob a ótica dos resultados do Enem. Rev. Bras. Ensino Fís., v. 40, n. 4, p. e4402, 2018.

BRASIL. Matriz de Referência Enem. Brasilia DF, 2015. Disponível em: < http://download.inep.gov.br/download/enem/matriz_referencia.pdf >. Acesso em: 25 mar 2020.

. Curso Técnico de Nível Médio em Química na Forma Integrada Regime Integral: Plano de Curso. Macapá AP, 2016. Disponível em: < https://portal.ifap.edu.br/index.php/publicacoes/item/1100-resolucao-n-20-2018consup >. Acesso em: 27 abril 2019.

$\mathrm{RC}: 78536$

Disponível em: https://www.nucleodoconhecimento.com.br/formazione-it/fisica- 
. Expansão da Rede Federal. Rede Federal de Educação Profissional, Científica e Tecnológica. 2018. Disponível em: < http://redefederal.mec.gov.br/expansao-da-rede-federal >. Acesso em: 27 abril 2019. . Curso Técnico em Química - Integrado - Campus Macapá. Macapá AP, 2019. Disponível em: http://www.ifap.edu.br/index.php/component/content/article?id=398 >. Acesso em: 27 abril 2019.

CASTRO, G. N. V. et al. Análise de Eficiência Acadêmica dos cursos subsequentes, nas modalidades à distância e presencial, ofertados pelo Instituto Federal do Amapá (2018). Research, Society and Development, v. 9, n. 8, p. e208985262, 2020. https://rsdjournal.org/index.php/rsd/article/view/5262

GASPAR, A. Cinqüenta anos de ensino de Física: Muitos equívocos, alguns acertos e a necessidade do resgate do papel do professor $\underline{X V \text { Encontro de }}$ Físicos do norte e Nordeste Natal RN 1997.

MARQUES, J. D. C. et al. Nível Médio Técnico e Cursos de Graduação: comparativo de vagas e ingressantes no Instituto Federal do Amapá, Brasil (20172018). Research, Society and Development, v. 9, n. 8, p. e228985375, 2020 https://rsdjournal.org/index.php/rsd/article/view/5375

PACHECO, E. M. Os Institutos Federais: Uma Revolução na Educação Profissional e Tecnológica. Natal RN: IFRN, 2010. 28p.

PENHA, A. C. F. M. et al. Matrículas da Educação Especial na Educação Profissional Técnica de Nível Médio no Estado do Amapá (2015-2018). Research, Society and Development, v. 9, n. 7, p. e881974867, 2020. https://rsdjournal.org/index.php/rsd/article/view/4867

$\mathrm{RC}: 78536$

Disponível em: https://www.nucleodoconhecimento.com.br/formazione-it/fisicaallenem 
PIRES, M. A.; VEIT, E. A. Tecnologias de Informação e Comunicação para ampliar e motivar o aprendizado de Fisica no Ensino Medio. Revista Brasileira de Ensino de Fısica, v. 28, n. 2, p. $241-248,2006$.

RIBEIRO, J. L. P.; VERDEAUX, M. D. F. D. S. Atividades experimentais no ensino de óptica: uma revisão. Revista Brasileira de Ensino de Fısica, v. 34, n. 4, p. 110, 2012.

SBF. Carta aberta ao Inep quanto à adoção do Exame Nacional do Ensino Médio como critério de ingresso nas Universidades. São Paulo SP, 2011. Disponível em: < http://www.sbfisica.org.br/v1/arquivos_diversos/noticias/maio2014/carta-aoINEP.pdf >. Acesso em: 05 fev 2021.

SILVA, J. R. N. D.; ARENGHI, L. E. B.; LINO, A. Porque inserir física moderna e contemporânea no ensino médio? Uma revisão das justificativas dos trabalhos acadêmicos. R. B. E. C. T., v. 6, n. 1, p. 1-15, 2013.

SILVEIRA, F. L.; BARBOSA, M. C. B.; SILVA, R. Exame Nacional do Ensino Médio (ENEM): Uma análise crítica Rev. Bras. Ensino Fís., v. 37, n. 1, p. 1101, 2015.

SILVEIRA, F. L.; STILCK, J.; BARBOSA, M. Comunicações: Manifesto sobre a qualidade das questões de Física na Prova de Ciências da natureza no Exame Nacional de Ensino Médio. Caderno Brasileiro de Ensino de Física, v. 31, n. 2, p. 473-479, 2014.

TOTI, F. A.; PIERSON, A. H. C. Elementos para uma aproximação entre a física no ensino médio e o cotidiano de trabalho de estudantes trabalhadores. Investigações em Ensino de Ciência, v. 15, n. 3, p. 527-552, 2010

Pubblicato: Marzo 2021

Approvato: Marzo 2021

$\mathrm{RC}: 78536$

Disponível em: https://www.nucleodoconhecimento.com.br/formazione-it/fisica- 